\title{
Challenges in the Field of Biomedical Engineering Research
}

\author{
Neeraj Sharma* \\ School of Biomedical Engineering, Indian Institute of Technology, India
}

Received: 眥 March 19, 2018; Published: 眥 March 29, 2018

*Corresponding author: Neeraj Sharma, Professor, School of Biomedical Engineering, Indian Institute of Technology, Banaras Hindu University, Varanasi, India, Tel: 9235633730; Email: neeraj.bme@itbhu.ac.in

\section{Editorial}

Biomedical Engineering is the most recent and challenging field of engineering that involves applying the principles, knowledge and approaches from all major fields of sciences and engineering to provide medical/clinical experts with the accurate tools for diagnostics, clinical studies and treatment. Biomedical Engineering in itself is a highly research oriented and interdisciplinary field. Biomedical engineering derives its principle and knowledge from all major engineering and science branch such as electronic, electrical, instrumentation, mechanical, computers etc. and chemistry, physics, nuclear physics, biology, pharmacology, physiology, etc. Whenever some new technology is developed its application in biomedical engineering is always searched. For example, the development of optical fibers in late 1980s has led to the development of endoscope indeed a useful device for abdomen examination and surgery applications. Further, most of the high end imaging systems such as CT scan, MR imaging have been only possible because of tremendous advancement in computer technology both at the hardware and software end. The list is long and it is not possible to cover all these in the present article. Finally, we can say that it is only because of technological advancement that we have been successfully able to design and develop accurate diagnostic devices (Ultra sound scan, CT scan, ECG, EMG and EEG machines etc.), prosthetic devices (pacemaker, heart valves, artificial limbs), treatment and surgery devices (LASER based surgery systems, electrosurgical units, radiotherapy systems, Lithotripters etc). Thus, advancement in the field of science and technology from all fronts has contributed a lot to the research and development of biomedical engineering.

Further from biomedical engineering point of view, some of the main research areas are as follows:

Further, from biomedical engineering point of view researchers must focus on following practical tips while carrying out research (Table 1).
Table 1.

\begin{tabular}{|c|c|}
\hline Biomedical Instrumentation & Clinical Engineering \\
\hline Biosensors \& Bio MEMs & $\begin{array}{c}\text { Computer-Integrated \& Computer- } \\
\text { Assisted Surgery }\end{array}$ \\
\hline Bio-signal Processing & Controlled Drug Delivery \\
\hline Biomaterials & Medical Robotics \\
\hline Physiological System Modeling & Micro Nano Biomedical devices \& \\
\hline systems
\end{tabular}

a) Biomedical researcher must understand the biological problem clearly for which solution is needed and this must be done in consultation with medical/clinical experts. A detailed research/experimental protocol must be built and same should be approved by expert committee (particularly for ethical clearance).

b) A base study may be conducted to evaluate and compare the existing methods and techniques that best address the problem. In case there are limitations in the existing technology research may be conducted to propose new and better solutions.

c) Careful selection of biomedical sensor: Biomedical sensor is used extensively for biomedical research to pick up biomedical signals and other physiological parameters thus play an important role in number of diagnostic and medical 
applications. Hence, there is strong need for accurate selection of biomedical sensor for a particular problem.

d) Biomedical signal (data) acquisition and processing strategy: Biomedical signals are of very low amplitude typically in the range of few micro volts to few milli volts and if not handled properly can easily get corrupted by noise or artifacts. The presence of noise in biomedical signal has significant effect and can suppress the relevant information.

e) In some situation it is advisable to build mathematical or hardware model of the physiological/biological system to be studied and same can be analyzed through simulation experiments further, in case study included in the present chapter mathematical modeling of dynamic cerebral auto regulation system and its simulation procedure has been demonstrated. Simulation studies are helpful in building up experimental design at first stage without going for actual testing or trial on the physiological system, for example testing of the defibrillator device is mainly carried out on simulator which may be otherwise dangerous to be tested on actual subject.

\section{Conclusion}

Biomedical Engineering in itself is a highly research oriented and interdisciplinary field and combines engineering with medical and biological sciences to improve healthcare diagnosis and treatment. There is definitely a set procedure to carry out research in biomedical engineering aiming at design and development of reliable health care technology.
(C) (P) This work is licensed under Creative To Submit Your Article Click Here: Submit Article

DOI: $10.32474 /$ OAJBEB.2018.02.000128

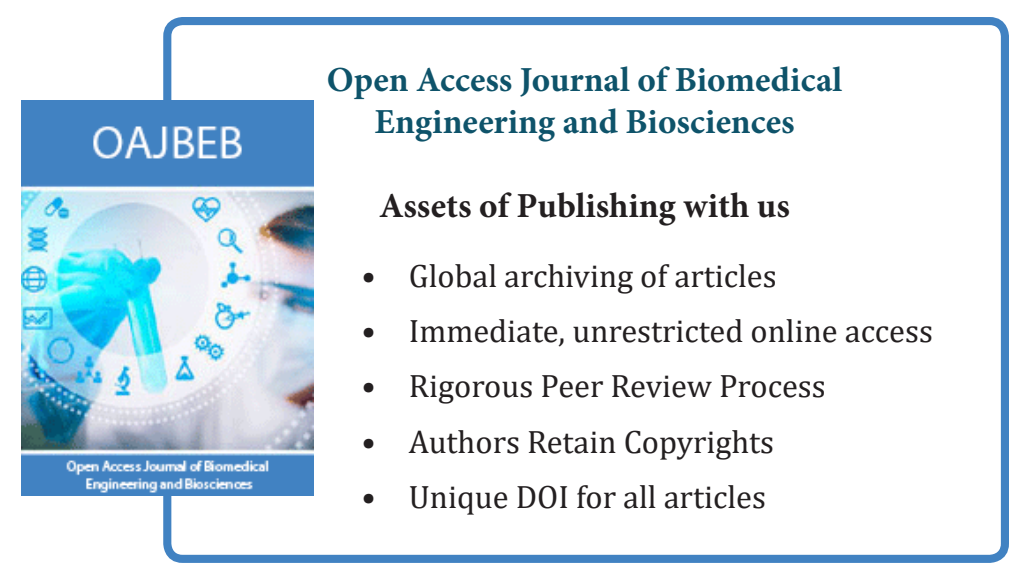

